\title{
Indicadores de sostenibilidad urbana para la ciudad de \\ Cuenca-Ecuador: construcción sostenible de edificaciones
}

\section{Urban sustainability indicators for the city of Cuenca-Ecuador: sustainable construction of buildings}

1 Paola Alejandra Ortiz Benavides https://orcid.org/0000-0001-7812-903X

Universidad Católica de Cuenca, Maestría en Construcciones con mención en Administración de la Construcción Sustentable, Azuay, paola.ortiz.75@est.ucacue.edu.ec

2 Juan Felipe Quesada Molina https://orcid.org/0000-0002-6931-0192 Universidad Católica de Cuenca, Maestría en Construcciones con mención en Administración de la Construcción Sustentable, Azuay felipe.quesada@ucacue.edu.ec

Artículo de Investigación Científica y Tecnológica

Enviado: $10 / 12 / 2021$

Revisado: $27 / 12 / 2021$

Aceptado: $17 / 01 / 2022$

Publicado:05/03/2022

DOI: https://doi.org/10.33262/concienciadigital.v5i1.2.2088

Cítese: $\quad$ Ortiz Benavides, P. A., \& Quesada Molina, J. F. (2022). Indicadores de sostenibilidad urbana para la ciudad de Cuenca-Ecuador: construcción sostenible de edificaciones . ConcienciaDigital, 5(1.2), 105-125. https://doi.org/10.33262/concienciadigital.v5i1.2.2088

CONCIENCIA DIGITAL, es una Revista Multidisciplinar, Trimestral, que se publicará en soporte electrónico tiene como misión contribuir a la formación de profesionales competentes con visión humanística y crítica que sean capaces de exponer sus resultados investigativos y científicos en la misma medida que se promueva mediante su intervención cambios positivos en la sociedad. https://concienciadigital.org La revista es editada por la Editorial Ciencia Digital (Editorial de prestigio registrada en la Cámara Ecuatoriana de Libro con No de Afiliación 663) www.celibro.org.ec 


\section{Palabras} claves: Indicadores, construcción sostenible, área urbana, evaluación.

Keywords: Indicators, sustainable construction, urban area, evaluation
Resumen

Introducción. En el Ecuador, la explosión urbana y sus escenarios de inequidad plantean nuevos desafíos como: recuperar el suelo urbano, generar vivienda adecuada y mitigar impactos ambientales, que brinden a los habitantes confort y un ambiente saludable. En la provincia del Azuay, el 50\% de las familias no poseen vivienda o habitan en viviendas inadecuadas con problemas como la precariedad de los materiales usados en la construcción, falta de servicios básicos, emplazamiento de viviendas en lugares de alto riesgo, mala calidad del aire, entre otros. En las últimas dos décadas el promedio de crecimiento anual de la huella urbana de algunas ciudades latinoamericanas ha sido del 3-4\%; entre estás, Cuenca es la tercera ciudad con la aglomeración urbana más grande del Ecuador. Objetivo Evaluar un marco de Indicadores Sostenibles Urbanos propuestos en base a los resultados obtenidos del análisis de las herramientas y metodologías internacionales más relevantes. Metodología. El nivel de investigación es descriptivo, el diseño de investigación fue cualitativo, de tipo prospectivo y transversal. Se analizó la bibliografía más relevante sobre el tema, se obtuvo un conjunto de indicadores validados por juicio de expertos. Resultados. El documento final contribuirá a la comunidad con un conjunto de Indicadores Sostenibles que se puedan aplicar en el contexto local, los que se desprenden de un proceso de determinación, selección, validación y aplicación de indicadores universales. Conclusión. Se concluyó que, en la ciudad de Cuenca, no existe prioridad para que la construcción sostenible se desarrolle, uno de los aspectos que influye, es la falta de políticas claras e incentivos para que los stakeholders consideren en sus proyectos todos los procesos, que se involucran durante el ciclo de vida de una edificación sostenible.

\section{Abstract}

Introduction. In Ecuador, the urban explosion presents scenes of inequity, which poses new challenges such as recovering urban land, generating adequate housing, and mitigating environmental impacts to provide the inhabitants with comfort and a healthy environment. In Azuay province, 50\% of families either do not own a home or dwell in inadequate housing with problems involving vulnerability and riskiness of the materials used for construction, lack of basic services, housing developing in high-risk areas, or 
poor air quality, among others. In the last two decades in some Latin American cities, the average annual growth of the urban footprint has been 3-4\% while in Ecuador; Cuenca is the third city with the largest metropolitan area. Objective. Assess a framework of Sustainable Urban Indicators proposed based on the results from the analysis of the most relevant international techniques and methodologies. Methodology. The level of research is descriptive, the research design was qualitative, prospective and cross-sectional. The most relevant bibliography on the subject was analyzed, a set of indicators validated by expert judgment was obtained. Results. The final document, containing a set of Sustainable Indicators that can be applied to the local context and which come from a process of determination, selection, validation and application of universal indicators, will be a contribution to the community. Conclusion. It was concluded that, in the city of Cuenca, there is no priority for sustainable construction to develop, one of the aspects that influences it is the lack of clear policies and incentives for stakeholders to consider all processes in their projects, which are involved during the life cycle of a sustainable building.

\section{Introducción}

\section{Antecedentes}

El crecimiento acelerado de la mancha urbana de las ciudades, está generando retos importantes y complejos en el camino a lograr un desarrollo sostenible, obliga a estas a contar con espacios de planificación urbana efectiva (Terraza et al., 2014), en este sentido la comunidad científica internacional propuso varias herramientas de evaluación y guías metodológicas basadas en indicadores que miden, monitorean y evalúan el progreso de las ciudades hacia la sostenibilidad, uno de los puntales principales para lograr este objetivo, es fomentar el emplazamiento de construcciones que sean eficaces y eficientes desde su concepción y diseño. La vivienda, ya sea como capital tangible, activos o méritos, debe utilizarse para garantizar las necesidades de vida de los diferentes hogares a todo nivel. Asimismo, la condición de un activo excede su alcance físico (construcción) e involucra todos los aspectos de la determinación de su entorno y ubicación relativa (Terraza et al., 2014).

Este enfoque, da las pautas para considerar a las edificaciones como un instrumento importante para la transformación de los barrios urbanos; por tanto, se hace imperativo conocer un marco de indicadores locales que sirvan para evaluar el desempeño de las 
construcciones en todas las etapas del ciclo de vida, pues estas contribuyen con elevados consumos de agua, electricidad y generación de residuos que ocasionan impactos negativos al medio ambiente, en respuesta a esta problemática, este trabajo está dirigido al aporte de un marco de Indicadores Sostenibles Urbanos locales, destinados a evaluar las construcciones con relación al impacto que generan en el área urbana de la ciudad.

Sin embargo, conocemos ¿cuáles son los Indicadores Sostenibles apropiados para medir la eficacia y eficiencia de las construcciones emplazadas en el área urbana, que aporten al desarrollo sostenible de la ciudad de Cuenca?, es por ello, que el objetivo principal de esta investigación es evaluar un marco de indicadores, con base en los resultados obtenidos del análisis de las herramientas y guías metodológicas más relevantes a nivel internacional y proponer indicadores que sean factibles de aplicarlos en la localidad.

\section{Marco teórico, breve historia y ciudades sostenibles}

En las ciudades viven unos 3.500 millones de personas, esta cifra representa la mitad de la humanidad y seguirá en aumento (Organización de las Naciones Unidas [ONU], 2015), en los próximos decenios, el $95 \%$ de la expansión urbana tendrá lugar en países en desarrollo, como consecuencia, las ciudades se enfrentan a la formación de barrios marginales con aproximadamente 828 millones de personas que los habitan, desde este punto de vista, el "desarrollo sostenible" es objeto de preocupación a nivel internacional (ONU, 2000).

$\mathrm{Al}$ respecto, nos retrotraemos hacia donde nació el término "desarrollo sostenible", el cual se acogió dentro del informe publicado en 1987, elaborado por la Comisión Brundtland, para las Naciones Unidas, con el Título original "Our Common Future", informe que define "está en manos de la humanidad hacer que el desarrollo sea sostenible, duradero, o sea, asegurar que satisfaga las necesidades del presente sin comprometer la capacidad de las futuras generaciones para satisfacer las propias" (Commission on Environment and Development, 1987).

Así mismo, en 1992 en la Cumbre de la Tierra organizada por la ONU y celebrada en Río de Janeiro en Brasil, la Declaración de Río sobre Medio Ambiente y Desarrollo formaliza el concepto de "desarrollo sostenible" como "un desarrollo que responda a las necesidades del presente al ritmo de la renovación de los recursos, es decir, que no comprometa el de las generaciones futuras" (Cumbre de la Tierra, 1992).

Con estas definiciones puestas sobre la mesa, el Consejo Internacional de Iniciativas Ambientales Locales, ICLEI 2015, propuso el siguiente concepto de desarrollo sostenible como "aquel que ofrece servicios ambientales, sociales y económicos básicos a todos los miembros de una comunidad sin poner en peligro la viabilidad de los sistemas naturales, construidos y sociales de los que dependen la oferta de estos servicios" (Bustos y Chacón, 
2009), aquí nacen las tres dimensiones: medioambiental, social y económico, por consiguiente, las ciudades deben apuntalarse en los tres ejes, pues son interdependientes y no alcanzarán la sostenibilidad si intervienen de manera autónoma.

Con la finalidad de abordar la problemática ambiental y de desarrollo, posteriormente, en 2015 todos los Estados Miembros de las Naciones Unidas aprobaron 17 Objetivos de Desarrollo Sostenible (ODS) como parte de la Agenda 2030, entre estos resaltamos el Objetivo 11 cuya meta es "Lograr que las ciudades sean más inclusivas, seguras, resilientes y sostenibles."

\section{Construcción sostenible y sistemas de evaluación}

Con el crecimiento de los asentamientos humanos que se emplazan en las áreas urbanas para acceder a mejores servicios, las ciudades ejercen una fuerte carga sobre el ecosistema, por esto, se han cuestionado las tramas urbanas y los crecimientos dispersos de las ciudades, pues son las principales causantes de las emisiones globales de gases efecto invernadero (GEI) en un 80\% (Valdez, 2021). Es por esto que, el concepto de ciudades sostenibles está ganando notoriedad, buscando una respuesta a esta problemática (Bibri, 2018). Según Banco Interamericano de Desarrollo (BID, 2014) una ciudad sostenible es aquella que brinda a sus habitantes una buena calidad de vida, minimiza los impactos ambientales, resguarda su entorno natural y físico, adicionalmente es manejada por un gobierno local con la capacidad de mantener el crecimiento económico y urbano involucrando para ello a la ciudadanía.

En este contexto, los usuarios actuales, son más exigentes y analizan con mayor rigor el proceso para la obtención de los productos y los métodos seguidos para la conservación del medio ambiente, demandando a los gobiernos la creación de normativas que encaminen a la ciudad a buscar un desarrollo de la construcción sostenible, ya que la demanda de estas ha crecido considerablemente en la industria de la arquitectura (Horman et al., 2006). La construcción sostenible se enfoca en el conjunto de acciones positivas que influyan en su ciclo de vida, considerando la reducción de los impactos negativos en el medio ambiente (Serrano et al., 2015).

Actualmente, más de 150 países efectúan evaluaciones de sustentabilidad, contando con más de 50 metodologías establecidas, en los años 90 algunos países empezaron a evaluar cuantitativa y cualitativamente sus edificios (Soffia, 2020), entre las herramientas de evaluación que tienen mayor influencia internacionalmente existen: BREEAM, LEED, VERDE, CASBEE y Qualitel (Quesada Molina, 2014), que analizan los aspectos más relevantes de una edificación como son agua, materiales, energía, entre otros (Soffia, 2020); sin embargo, los investigadores encuentran dificultades en aplicarlas en regiones diferentes de las que fueron elaboradas, existen varios enfoques y métodos de evaluación 
que se han basado en Indicadores Sostenibles, que miden y monitorean los proceso de construcción para así conseguir el éxito deseado (Quesada Molina, 2014).

ONU-Hábitat plantea Indicadores Sostenibles para evaluar que una vivienda sea adecuada y durable, entre estos tenemos: tenencia, servicios básicos, materiales, asequibilidad, habitabilidad y ubicación (UN-Hábitat et al., 2018), varios países implementaron algunos de estos indicadores internacionales, más en algunos casos se encontraban desarrollando y aplicando indicadores propios (Quiroga, 2001).

Los Indicadores son herramientas concretas que guían en los procesos de diseño y evaluación de la política pública para impulsar a los países hacia el desarrollo sostenible (Quiroga, 2001); además de cuantificar y simplificar la información tienen otras características que los definen, por tanto, deben ser: útiles, pertinentes, transmitir información significativa, reflejar las metas planteadas, ser relevantes para las políticas públicas y de fácil lectura; sin embargo, diferentes investigadores consideran importante el mantenimiento y actualización periódica de los Indicadores Sostenibles, pues muchas veces no reflejan la realidad de un lugar o comunidad (Batalhao et al., 2019).

\section{Desarrollo local sostenible}

La Constitución de la República del Ecuador, en la Sección VI, Hábitat y vivienda, reconoce el derecho de los ecuatorianos a un ambiente seguro y saludable y una vivienda adecuada y digna, por esta razón se han aprobado leyes, acuerdos e incentivos encaminados a promover la sostenibilidad, como por ejemplo la Ley Orgánica de Eficiencia Energética y Punto Verde del Ministerio del Ambiente, en ese sentido, se generó un interés de los Gobiernos Autónomos Descentralizados (GAD) al crear y aprobar ordenanzas como la del Municipio de Quito basada en el Plan Metropolitano de Desarrollo y Ordenamiento Territorial, en proyectos eco-eficientes.

Al respecto y al ser la tercera ciudad con la aglomeración urbana más grande del Ecuador (Terraza et al., 2014), el Gobierno Autónomo Descentralizado Municipal de Cuenca, desde mayo de 1997, creó la Comisión de Gestión Ambiental (C.G.A.), una entidad pública descentralizada que coordina y lidera la gestión ambiental y ejerce las competencias de Autoridad Ambiental de Aplicación Responsable, si bien es cierto no emite una certificación, la entidad otorga la Licencia Ambiental en donde se establece la obligatoriedad del cumplimiento de la normativa ambiental.

En cuanto a la existencia de edificaciones emplazadas en la ciudad de Cuenca, que ostenten algún tipo de certificación sustentable, son seis edificaciones las que están en proceso o ya cuentan con una certificación, como se muestra en la tabla 1 / figura 1, emplazadas en las parroquias urbanas Yanuncay, San Sebastián y Machángara, que representa el $0,00002 \%$ de los 375.184 bloques construidos aproximados (no se 


\section{Conciencia}

consideran los bloques en régimen de propiedad horizontal) emplazados en el área urbana (dato derivado de la documentación entregada por la Dirección de Avalúos y Catastros del GAD de Cuenca).

Tabla 1

Construcciones sostenibles emplazados en el área urbana de Cuenca

\begin{tabular}{ll}
\hline \multicolumn{1}{c}{ Proyecto } & \multicolumn{1}{c}{ Certificación } \\
\hline Campus Bicentenario de la Universidad del Azuay. & Certificación EDGE Advanced \\
Unidad Educativa. & \\
Edificio Häuser. Apartamentos. & Certificado EDGE preliminar de GBCI \\
Río Azul. Edificio residencial. & Certificación Final EDGE de GBCI \\
Supermaxi Challuabamba. & Certificación en curso LEED BD+C: Retailv4 - \\
Comisariato de alimentos. & LEED v4 \\
Goya. Complejo de apartamentos. & Certificado EDGE preliminar de GBCI \\
Cumbres De Loretto. Condominios. & Certificado EDGE preliminar \\
\hline
\end{tabular}

Fuente: EDGE BUILDINGS / U.S. Green Building Council

\section{Figura 1}

Edificaciones que están en proceso o cuentan con el certificado de sostenibilidad

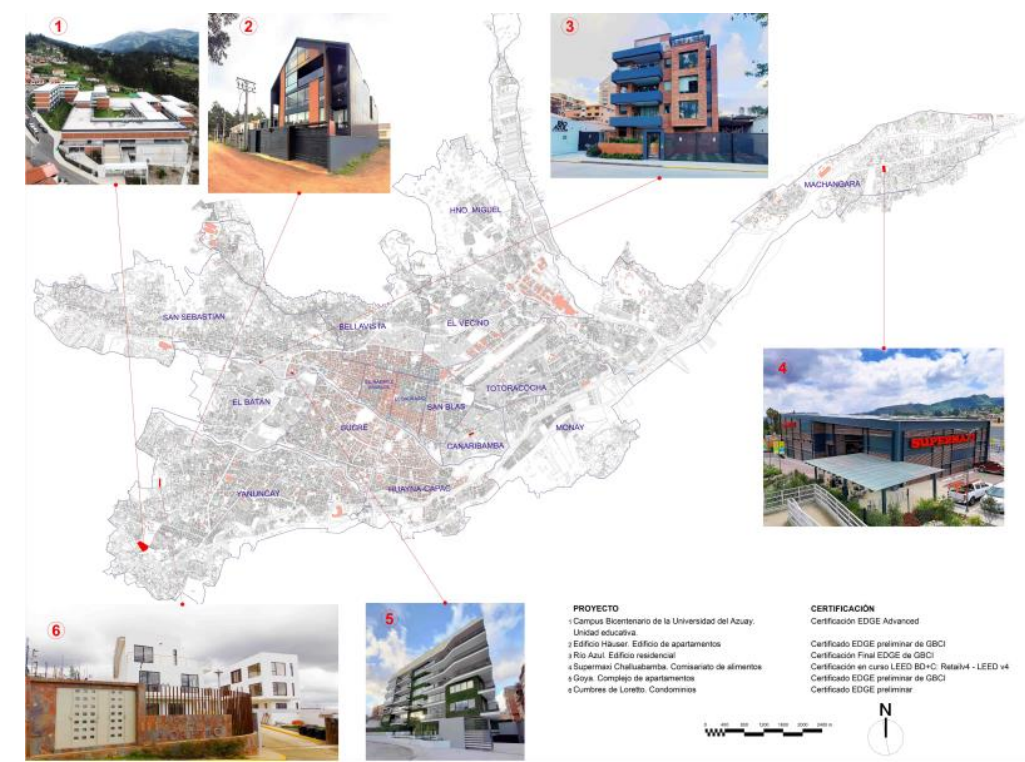

Fuente: Plano de la Dirección de Planificación del GAD de Cuenca y complementado 


\section{Metodología}

El nivel de investigación es descriptivo, el diseño de investigación fue cualitativo, de tipo prospectivo y transversal, se establecieron tres etapas: Etapa1- Identificar, definir y homologar los indicadores; Etapa 2- Validar el contenido por Juicio de expertos; Etapa 3- Aplicar los indicadores en un caso de estudio determinado, como se muestra en la figura 2.

Figura 2

Esquema metodológico de investigación

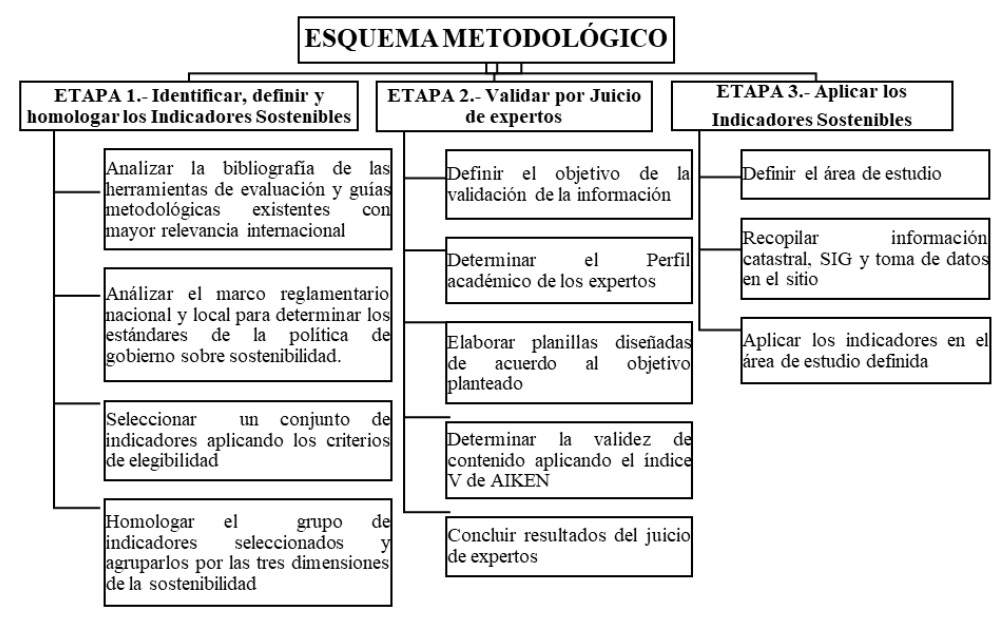

Para obtener el conjunto final de indicadores, se consideraron los siguientes pasos y componentes: revisión preliminar, elegir resultados y metas, seleccionar indicadores, establecer línea base, seleccionar objetivos, recopilar datos, analizar resultados e identificar deficiencias, reportar hallazgos, aplicar hallazgos (Verma \& Raghubanshi, 2018).

\section{ETAPA 1.- Identificar, definir y homologar los Indicadores Sostenibles Urbanos}

En esta etapa se identificó y definió los datos resultantes del análisis de la bibliografía de las herramientas de evaluación y guías metodológicas, se consideraron los siguientes criterios de categorización: Claridad, disponibilidad de métodos e información y que abarquen varias áreas de las funciones de la ciudad (Ahvenniemi et al., 2017).

La bibliografía analizada fue la siguiente: tres herramientas de evaluación y certificación, que son: BREEAM COMMUNITIES 2012, CASBEE FOR CITIES 2012, LEED v4.1. 2020; así mismo, tres Guías metodológicas, que son: Iniciativa Ciudades Emergentes y Sostenibles y su Anexo de Indicadores, Norma Internacional ISO 37120 Desarrollo Sostenible de Comunidades y los Objetivo de Desarrollo Sostenible (ODS) enfocados en el Objetivo 11. 
Del análisis realizado y aplicando los criterios de elegibilidad de las características intrínsecas de los indicadores, que deben ser relevantes, pertinentes, claros, medibles, verificables y existir disponibilidad de datos, se realizó la homologación de los indicadores.

\section{ETAPA 2.- Juicio de expertos}

Este método de validez de contenido es utilizado para cotejar la confiabilidad de una investigación, se define como un dictamen de expertos que tienen una trayectoria en el tema planteado y pueden aportar información, juicio y valoraciones (Escobar y Cuervo, 2008).

Con relación a los expertos fueron seleccionados con base al siguiente perfil: Arquitectos, Ingenieros o profesionales de ramas afines, preferentemente con Maestría en Ordenación Territorial, Maestría en Urbanismo, Territorio y Paisaje, Maestría en Construcciones, Maestría en Gestión y Administración del Patrimonio Cultural, Maestría en Diseño Ambiental, Certificadores para edificios sustentables, con una experiencia mínimo de 5 años en el ámbito privado o público. Para la validación de contenido se requirió de diez expertos nacionales, siendo un número que brinda una estimación confiable para la validación de contenido (Escobar y Cuervo, 2008).

El objetivo del Juicio de expertos, fue validar con base a su pericia los datos propuestos de: Categorías, Indicadores Sostenibles y objetivos, para la recolección de datos se elaboró una planilla, diseñada de acuerdo al objetivo trazado, de fácil comprensión y uso, para la evaluación de los indicadores se planteó los criterios de suficiencia, claridad, coherencia y relevancia y para la calificación se establecieron cuatro niveles que son: 1. No cumple con el criterio, 2. Bajo Nivel, 3. Moderado nivel y 4. Alto nivel (Escobar \& Cuervo, 2008), para cuantificar la validez de contenido se utilizó el coeficiente V de Aiken (Galicia et al., 2017; Soto \& Segovia, 2009), como se muestra en la figura 3.

\section{Figura 3}

\section{Planilla de evaluación elaborada por un experto}

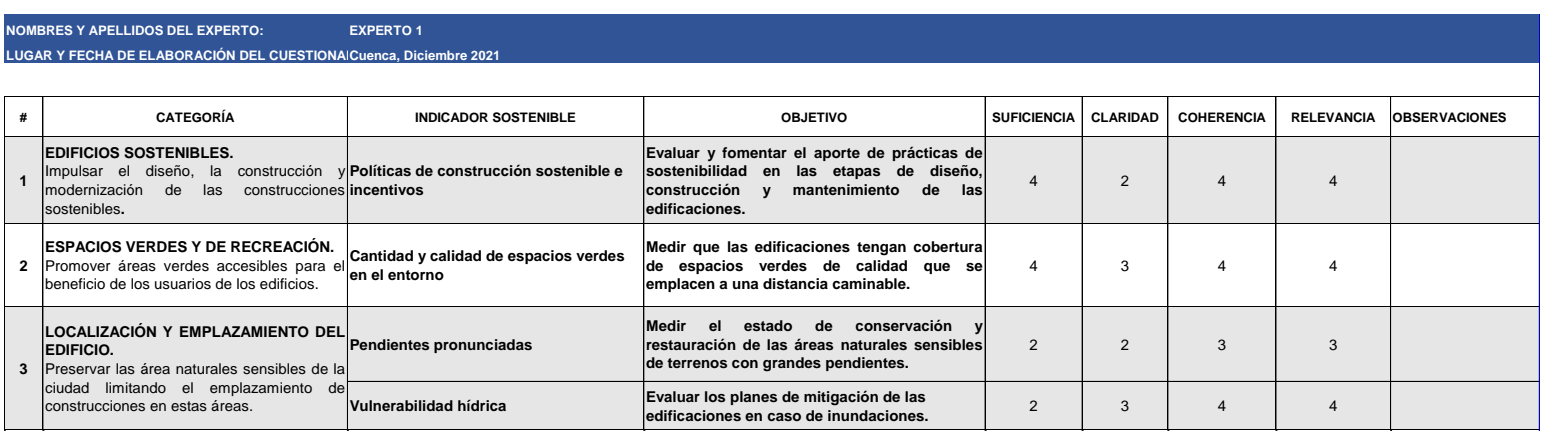


ETAPA 3.- Aplicar los indicadores propuestos en área de estudio definida

El alcance de este trabajo es el área urbana de la ciudad de Cuenca, capital de la provincia del Azuay, está formada por 15 parroquias urbanas y 21 rurales, Cuenca en términos de población es la tercera ciudad más grande del Ecuador, en el 2010 el INEC realizó una proyección de los habitantes para el 2020 de 636.996 habitantes (Instituto Nacional de Estadísticas y Censos [INEC], 2010). Con la finalidad de seleccionar un espacio geográfico se tomó las siguientes consideraciones: ocupación del suelo, trama urbana, disponibilidad de información, para la evaluación se tomó, tres edificaciones emplazadas en las parroquias urbanas Cañaribamba, Totoracocha y Yanuncay.

\section{Resultados}

\section{Análisis de la bibliografía internacional}

Del análisis de las herramientas y guías metodológicas se seleccionó un total de cuarenta indicadores pertinentes al tema, distribuidos en un número de trece indicadores en LEED V4.1, trece indicadores en BREEAM COMMUNITIES 2012, tres indicadores en CASBEE FOR CITIES 2012; y, dos indicadores en los Objetivos de Desarrollo Sostenible, tres en la Norma Internacional ISO 37120 y seis en la Iniciativa Ciudades Emergentes y Sostenibles, como se muestra en la Tabla 2.

Tabla 2

Indicadores seleccionados del análisis de la bibliografía de las herramientas de evaluación

\begin{tabular}{ll}
\hline \multicolumn{1}{c}{ LEED v4.1. abril 2020 } & \multicolumn{1}{c}{ BREEAM COMMUNITIES 2012 } \\
\hline $\begin{array}{l}\text { Calidad de vida (QL) } \\
\text { Crédito: Vivienda Asequible }\end{array}$ & Paso 1. Bienestar social y económico \\
\hline Eficiencia del agua (WE) & SE 03 -Evaluación del riesgo de inundaciones \\
Prerrequisito: Acceso y Calidad del Agua & SE 04 - Contaminación acústica \\
\hline Eficiencia del agua (WE) & Paso 1. Recursos y energía \\
Prerrequisito: Gestión Integrada del Agua & RE 01 - Estrategia energética \\
\hline Energía y gases efecto invernadero (EN) & \\
Prerrequisito: Acceso a la energía, Confiabilidad, & Paso 1. Recursos y energía \\
Capacidad de Resiliencia & RE 02 - Edificios e infraestructura existentes \\
\hline $\begin{array}{l}\text { Energía y gases efecto invernadero (EN) } \\
\text { Prerrequisito: Energía y Gestión de Emisiones de }\end{array}$ & Paso 1. Recursos y energía \\
Gases de Efecto Invernadero & RE 03 - Estrategia del agua \\
\hline
\end{tabular}




\section{Conciencia}

\section{Tabla 2}

Indicadores seleccionados del análisis de la bibliografía de las herramientas de evaluación (continuación)

LEED v4.1. abril 2020

Materiales y recursos (MR)

Crédito: Tratamiento de Residuos Orgánicos

Materiales y recursos (MR)

Prerrequisito: Manejo de Residuos Sólidos

Sistemas Naturales y Ecología (NS)

Crédito: Planificación de Resiliencia

Sistemas Naturales y Ecología (NS)

Crédito: Recursos Naturales, Conservación y

Restauración

Sistemas Naturales y Ecología (NS)

Prerrequisito: Espacios Verdes

Transporte y uso del suelo (TR)

Crédito: Uso Compacto, Mixto y Desarrollo

Orientado al Tránsito

Transporte y uso del suelo (TR)

Sitio de Alta Prioridad

Paso 3. Transporte y movimiento

TM 05 - Instalaciones de ciclismo

\section{BREEAM COMMUNITIES 2012}

Paso 2. Bienestar social y económico

SE 05 - Provisión de vivienda

Paso 2. Bienestar social y económico

SE 08 - Microclima

Paso 2. Transporte y movimiento

TM 04 - Acceso al transporte público

Paso 3. Bienestar social y económico

SE 15 - Diseño inclusivo

Paso 3. Recursos y energía

RE 04 - Edificios sostenibles

Paso 3. Recursos y energía

RE 06 - Eficiencia de recursos

Paso 3. Uso del suelo y ecología

Proceso Integrativo (IP)

LE 06 - Recolección de agua de lluvia

Créditos: Políticas de Construcción verde

\section{CASBEE FOR CITIES 2012}

Carga ambiental L). L1 Emisiones de CO2 de fuentes de energía. L1.3 Sector comercial

Q2 Aspectos sociales. Q2.1 Entorno de vida. Q2.1.1 Calidad adecuada de la vivienda

Q2 Aspectos sociales. Q2.1 Entorno de vida. Q2.1.4 Preparación para desastres

\section{OBJETIVO DE DESARROLLO SOSTENIBLE 11}

Indicador 11.1.1: Proporción de la población urbana que vive en barrios marginales, asentamientos informales o viviendas inadecuadas

Indicador 11.c.1 Proporción de apoyo financiero a los países menos adelantados que se asigna a la construcción y modernización de edificios sostenibles, resilientes y eficientes en el uso de recursos utilizando materiales locales. 


\section{Tabla 2}

Indicadores seleccionados del análisis de la bibliografía de las herramientas de evaluación (continuación)

\section{NORMA INTERNACIONAL ISO 37120}

13. Recreación

13.1 Metros cuadrados de espacio público recreativo cubierto por cápita (indicador de apoyo)

15. Refugio.

15.1 Porcentaje de la población urbana que vive en barrios marginales (indicador central)

7. Energía

7.3 Consumo de energía de los edificios públicos por año $\left(\mathrm{kWh} / \mathrm{m}^{2}\right)$ (indicador básico)

\section{INICIATIVA CIUDADES EMERGENTES Y SOSTENIBLES}

Sostenibilidad Ambiental y cambio climático. Tema: D. Energía.

SUBTEMA D.2 Eficiencia energética.

23. Existencia, monitoreo y cumplimiento de las normas sobre eficiencia energética

Sostenibilidad Ambiental y cambio climático. Tema: F. Mitigación del cambio climático.

SUBTEMA F.1 Sistemas de medición de emisiones de GEI

28. Existencia y monitoreo de un inventario de gases de efecto invernadero

Sostenibilidad Ambiental y cambio climático.

Tema: H. Vulnerabilidad ante amenazas naturales en el contexto del cambio climático

SUBTEMA H.2 Sensibilidad ante amenazas de origen natural

39. Infraestructura fundamental en situación de riesgo debido a construcción inadecuada o ubicación en zonas de riesgo no mitigable

Sostenibilidad Ambiental y cambio climático.

Tema: H. Vulnerabilidad ante amenazas naturales en el contexto del cambio climático

SUBTEMA H.2 Sensibilidad ante amenazas de origen natural

40. Porcentaje de hogares en riesgo debido a construcción inadecuada o ubicación en áreas con riesgo no mitigable

Sostenibilidad Urbana. Tema: I. Uso del suelo / Ordenamiento del territorio

SUBTEMA I.2 Vivienda. 43. Porcentaje de viviendas que no cumplen con los estándares de habitabilidad definidos por el país

Sostenibilidad Urbana. Tema: J. Desigualdad urbana. SUBTEMA J.2 Segregación socioespacial. 50.

Porcentaje de viviendas ubicadas en asentamientos informales

\section{Homologación de indicadores sostenibles seleccionados}

Con los indicadores seleccionados, se procedió a realizar la homologación, basada en las categorías y temas más significativos adaptados a la localidad, usando la técnica de 


\section{Conciencia}

elección por juicio, pues se establecieron según el criterio del investigador, con base en las herramientas y guías metodológicas y las características más relevantes, en este proceso se definieron trece Categorías las que agrupan a veinte y tres indicadores que fueron presentados a los expertos para validar su contenido, como se muestra en la Tabla 3.

\section{Tabla 3}

Indicadores homologados y agrupados por categorías

\begin{tabular}{|c|c|c|}
\hline$\#$ & CATEGORÍA & INDICADOR \\
\hline 1 & Edificios sostenibles & Políticas de construcción sostenible \\
\hline 2 & Espacios verdes y de recreación & $\begin{array}{l}\text { Cantidad y calidad de espacios verdes en el } \\
\text { entorno }\end{array}$ \\
\hline \multirow[t]{2}{*}{3} & Localización y emplazamiento del edificio & Pendientes pronunciadas \\
\hline & & Vulnerabilidad hídrica \\
\hline 4 & Planificación de resiliencia del edificio & Preparación para desastres \\
\hline \multirow[t]{2}{*}{5} & Movilidad y transporte & Proximidad a transporte público \\
\hline & & Instalaciones para bicicletas \\
\hline 6 & Uso de suelo & Conservación de Edificios Patrimoniales \\
\hline \multirow[t]{3}{*}{7} & Eficiencia del agua & Acceso y calidad del agua \\
\hline & & Estrategias para captar agua de lluvia \\
\hline & & Estrategia del agua \\
\hline \multirow[t]{4}{*}{8} & Energía y atmósfera & Acceso y calidad de la energía \\
\hline & & Consumo de energía de los edificios \\
\hline & & Estrategia energética \\
\hline & & Eficiencia energética \\
\hline \multirow[t]{3}{*}{9} & Manejo de residuos & Manejo de Residuos Sólidos \\
\hline & & Control de residuos hasta su recolección \\
\hline & & Tratamiento de Residuos Orgánicos \\
\hline \multirow[t]{2}{*}{10} & Accesibilidad y calidad de vivienda & Calidad adecuada de vivienda \\
\hline & & Viviendas inadecuadas \\
\hline 11 & Microclima & Ambiente exterior \\
\hline 12 & Materiales y recursos & Eficiencia de recursos. \\
\hline 13 & Diseño inclusivo & Diseño inclusivo \\
\hline
\end{tabular}




\section{Juicio de Expertos}

Con las evaluaciones de los jueces, se elaboró la matriz de datos, identificando a cada indicador con la letra I complementada con un número, tal como, I1, en la matriz se registró el nivel de calificación de cada pregunta por cada experto, como se muestra en la figura 4.

\section{Figura 4}

\section{Resultados del Juicio de expertos}
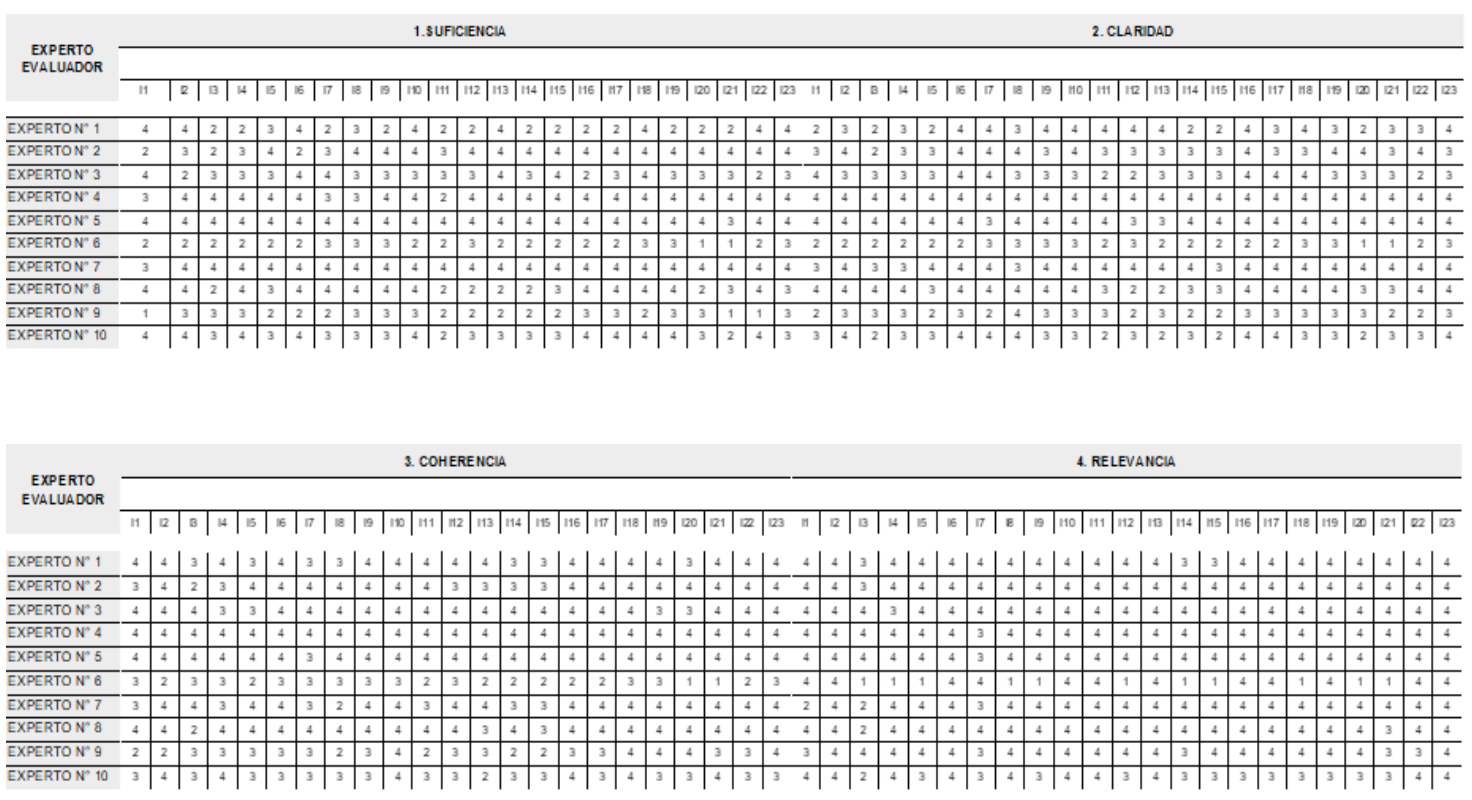

Para el cálculo del coeficiente de V de Aiken se utilizó la siguiente fórmula:

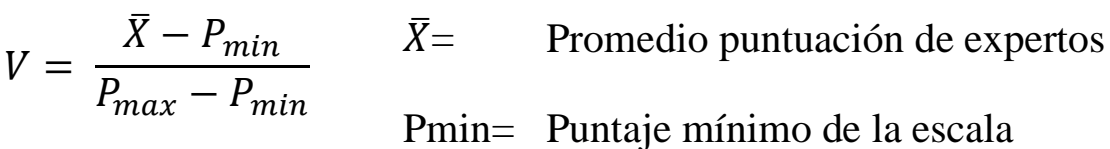

$$
\begin{aligned}
& \text { Pmax }=\text { Puntaje máximo escala }
\end{aligned}
$$

Este coeficiente puede obtener valores entre 0 y 1 , a medida que sea más elevado el valor computado, el ítem tendrá una mayor validez de contenido (Escurra, 1988).

Con los resultados de la evaluación del juicio de expertos y el procesamiento de los datos obtenidos, se proponen 12 Categorías y 19 indicadores cuyas características los convierten en aptos para aplicarlos en la localidad, de los cuales se estructuró la unidad de medida y los parámetros de evaluación, datos fundamentados en los criterios y métodos de evaluación de las herramientas LEED y BREEAM; Plan de Ordenamiento Territorial, Normas de la construcción de la localidad y los criterios emitidos por los expertos, como se muestra en la figura 5.

\section{Ciencia




\section{Figura 5}

Indicadores de sostenibilidad urbanos propuestos para la localidad, enfocados a la construcción sostenible de edificaciones

\begin{tabular}{|c|c|c|c|c|c|}
\hline \# & CATEGORÍA & INDICADOR & OBJETIVO & $\begin{array}{l}\text { UNIDAD } \\
\text { MEDIDA }\end{array}$ & MÉTODO DE EVALUACIÓN \\
\hline 1 & $\begin{array}{l}\text { EDIFICIOS SOSTENIBLES. } \\
\text { Impulsar el diseño, la construcción y modernización d } \\
\text { de las construcciones sostenibles. }\end{array}$ & $\begin{array}{l}\text { Obtención de un certificado } \\
\text { de construcción sostenible }\end{array}$ & $\begin{array}{l}\text { Evaluar y fomentar el aporte de prácticas de } \\
\text { sostenibilidad en las etapas de diseño, } \\
\text { construcción y mantenimiento de las } \\
\text { edificaciones. }\end{array}$ & Certificado & $\begin{array}{l}100 \% \text { de edificios en proceso o certificados } \\
\text { como edificios sostenibles. }\end{array}$ \\
\hline 2 & $\begin{array}{l}\text { ESPACIOS VERDES Y DE RECREACIÓN. } \\
\text { Promover áreas verdes accesibles para el beneficio e } \\
\text { de los usuarios de los edificios. }\end{array}$ & $\begin{array}{l}\text { Cantidad y calidad de } \\
\text { espacios verdes en el } \\
\text { entorno }\end{array}$ & $\begin{array}{l}\text { Medir que las edificaciones tengan cobertura de } \\
\text { espacios verdes de calidad que se emplacen a } \\
\text { una distancia caminable. }\end{array}$ & Distancia & $\begin{array}{l}90 \% \text { de las unidades de vivienda debe tener un } \\
\text { espacio verde como máximo a una distancia } \\
\text { caminable de media milla ( } 800 \text { metros). }\end{array}$ \\
\hline 3 & $\begin{array}{l}\text { PLANIFICACIÓN DE RESILIENCIA DEL EDIFICIO. P } \\
\text { Incorporar planes de resiliencia del edificio para tener } \\
\text { estrategias ante el daño potencial debido a los } \\
\text { peligros. }\end{array}$ & Preparación para desastres & $\begin{array}{l}\text { Evaluar la guía que contenga las estrategias } \\
\text { para afrontar los desastres naturales y las } \\
\text { medidas de recuperación con las que cuenta el } \\
\text { edificio. }\end{array}$ & Guía & $\begin{array}{l}\text { Guía de Planificación de Resiliencia del edificio } \\
\text { ante desastres. }\end{array}$ \\
\hline 4 & $\begin{array}{l}\text { MOVILIDAD Y TRANSPORTE. } \\
\text { Fomentar el uso de los diferentes sistemas de tr } \\
\text { transporte y la dotación de infraestructura para su } \\
\text { funcionamiento, implementando desde el diseño del } \\
\text { edificio criterios como distancia y calidad de los in } \\
\text { espacios. }\end{array}$ & $\begin{array}{l}\text { Proximidad a servicios de } \\
\text { transporte público } \\
\text { Instalaciones para bicicletas }\end{array}$ & $\begin{array}{l}\text { Medir que las edificaciones tengan cobertura de } \\
\text { transporte público con relación a la calidad y } \\
\text { distancia. } \\
\text { Medir que las edificaciones promuevan el uso de } \\
\text { transporte alternativo mediante la creación de } \\
\text { lugares adecuados para el almacenamiento de } \\
\text { las bicicletas. }\end{array}$ & Distancia & $\begin{array}{l}75 \% \text { de todos los edificios residenciales y no } \\
\text { residenciales existentes identificados se se } \\
\text { encuentran a una distancia a pie de } 1 / 4 \text { de milla } \\
\text { (400 metros) de un transporte público de calidad. } \\
\text { Estudios / viviendas de } 1 \text { dormitorio } \\
\text { estacionamiento para } 1 \text { bicicleta por cada dos } \\
\text { viviendas. Dimensiones } 2 \mathrm{~m} \text { de largo } \times 0,75 \mathrm{~m} \\
\text { de ancho } \\
\text { Viviendas de } 2 / 3 \text { dormitorios - estacionamiento } \\
\text { para } 1 \text { bicicleta. Dimensiones } 2 \mathrm{~m} \text { de largo } \times \\
0,75 \mathrm{~m} \text { de ancho }\end{array}$ \\
\hline 5 & $\begin{array}{l}\text { USO DE SUELO } \\
\text { Si el edificio tiene características históricas, deberá } \mathrm{P} \\
\text { conservar su identidad y pertenecer a un inventario } \\
\text { de los edificios patrimoniales de la ciudad. }\end{array}$ & $\begin{array}{l}\text { Conservación de Edificios } \\
\text { Patrimoniales }\end{array}$ & $\begin{array}{l}\text { Evaluar si las edificaciones de carácter } \\
\text { patrimonial constan dentro de un inventario y si } \\
\text { su grado de conservación es la óptima, en caso } \\
\text { de edificios restaurados medir si se ha } \\
\text { fomentado las prácticas de sostenibilidad. }\end{array}$ & $\%$ & $\begin{array}{l}100 \% \text { de edificios patrimoniales que estén } \\
\text { emplazados en un barrio histórico }\end{array}$ \\
\hline \multirow[t]{3}{*}{6} & \multirow{3}{*}{$\begin{array}{l}\text { EFICIENCIA DEL AGUA. } \\
\text { Promover el ahorro del agua dulce en el edificio, } \\
\text { mediante una correcta gestión del consumo del agua } \\
\text { potable, cobertura y reutilización de aguas lluvias. }\end{array}$} & Acceso y calidad del agua & $\begin{array}{l}\text { Medir si las edificaciones cuentan con un servicio } \\
\text { equitativo y de calidad del agua potable. }\end{array}$ & $\%$ & $\begin{array}{l}100 \% \text { de construcciones con cobertura de agua } \\
\text { potable. }\end{array}$ \\
\hline & & $\begin{array}{l}\text { Estrategias para captar y } \\
\text { recolectar agua de lluvia }\end{array}$ & $\begin{array}{l}\text { Valorar los sistemas de recolección y } \\
\text { reutilización del agua lluvia para uso sanitario, } \\
\text { riego de jardines y limpieza de la vivienda. }\end{array}$ & $\%$ & $\begin{array}{l}50 \% \text { de agua lluvia deberá ser utilizada para } \\
\text { actividades no potables }\end{array}$ \\
\hline & & Estrategia del agua & $\begin{array}{l}\text { Medir y valorar los diseños para minimizar el } \\
\text { consumo y la demanda operativa de agua de los } \\
\text { edificios, mediante la propuesta del uso de } \\
\text { artefactos de ahorro y control del agua. }\end{array}$ & Diseño & $\begin{array}{l}\text { Diseño enfocado a minimizar la demanda de } \\
\text { agua a través de la eficiencia }\end{array}$ \\
\hline \multirow[t]{2}{*}{7} & \multirow[t]{2}{*}{$\begin{array}{l}\text { ENERGíA Y ATMÓSFERA } \\
\text { Promover el acceso de la energía a todas las } \\
\text { construcciones, planificar desde el diseño estrategias } \\
\text { enfocadas a minimizar la demanda de la energía. }\end{array}$} & $\begin{array}{l}\text { Acceso y calidad de la } \\
\text { energía } \\
\text { Consumo de energía de los } \\
\text { edificios }\end{array}$ & $\begin{array}{l}\text { Medir si las edificaciones cuentan con acceso } \\
\text { seguro, fiable, resistente y equitativo a la } \\
\text { Medir el uso reducido y eficaz de energía. }\end{array}$ & $\begin{array}{c}\mathrm{k} \\
\mathrm{kWh} / \mathrm{m} 2 \mathrm{x} \\
\text { año }\end{array}$ & $\begin{array}{l}100 \% \text { de construcciones con cobertura de } \\
\text { energia. } \\
\text { Demanda energética } \mathrm{kWh} / \mathrm{m} 2 \text { por año }\end{array}$ \\
\hline & & Estrategia energética & $\begin{array}{l}\text { Medir y valorar los diseños para minimizar la } \\
\text { demanda operativa de energia y consumo de los } \\
\text { edificios, mediante la propuesta del uso de } \\
\text { dispositivos de ahorro energético, aislamiento, } \\
\text { materiales, iluminación natural. }\end{array}$ & Diseño & $\begin{array}{l}\text { Diseño enfocado a minimizar la demanda } \\
\text { operativa de energía y consumo. }\end{array}$ \\
\hline \multirow[t]{2}{*}{8} & \multirow{2}{*}{$\begin{array}{l}\text { MANEJO DE RESIDUOS. } \\
\text { Incentivar la reducción de la generación de basura, } \\
\text { implementando la gestión de los residuos sólidos y } \\
\text { orgánicos. }\end{array}$} & Manejo de Residuos Sólidos & $\begin{array}{l}\text { Evaluar si los edificios cumplen con la norma } \\
\text { INEN de separación de desechos }\end{array}$ & Norma & $\begin{array}{l}\text { Cumplimiento de norma INEN de separación de } \\
\text { desechos }\end{array}$ \\
\hline & & $\begin{array}{l}\text { Tratamiento de Residuos } \\
\text { Orgánicos }\end{array}$ & $\begin{array}{l}\text { Medir si los edificios cuentan con estrategias } \\
\text { para separar y tratar los residuos orgánicos de } \\
\text { otro tipo de residuos. }\end{array}$ & $\%$ & $\begin{array}{l}\text { Gestionar residuos para tratar } 75 \% \text { a } 100 \% \text { de } \\
\text { los residuos orgánicos. }\end{array}$ \\
\hline \multirow[t]{2}{*}{9} & \multirow[t]{2}{*}{$\begin{array}{l}\text { ACCESIBILIDAD Y CALIDAD DE VIVIENDA. } \\
\text { Posibilitar el acceso de la población a una vivienda } \\
\text { adecuada y que estén emplazadas en lugares } \\
\text { adecuados para mejorar su calidad de vida }\end{array}$} & Certificado de habitabilidad & $\begin{array}{l}\text { Evaluar si las edificaciones cuentan con } \\
\text { permisos de construcción, cumplimiento de áreas } \\
\text { mínimas y criterios mínimos de confort interior. }\end{array}$ & Normas & $\begin{array}{l}\text { Cumplimiento de normativas locales de la } \\
\text { construcción, áreas mínimas, iluminación, } \\
\text { ventilación. }\end{array}$ \\
\hline & & Viviendas inadecuadas & $\begin{array}{l}\text { Medir si los usuarios emplazan las edificaciones } \\
\text { en zonas consideradas como tugurios, } \\
\text { asentamientos informales y si las viviendas son } \\
\text { inadecuadas para tener una buena calidad de } \\
\text { vida. }\end{array}$ & $\%$ & $\begin{array}{l}\text { a) Hogares de tugurios (SH): }=100 \text { ([Número de } \\
\text { personas que viven en barrios } \\
\text { marginales]/Población de la ciudad) } \\
\text { b) Hogares de asentamientos informales (ISH): } \\
=100 \text { ([Número de personas que viven en } \\
\text { hogares de asentamientos informales]/ } \\
\text { Población de la ciudad) } \\
\text { c) Hogares con viviendas inadecuadas (IHH): } \\
=100 \text { ([Número de personas que viven en } \\
\text { viviendas inadecuadas] /Población de la ciudad) }\end{array}$ \\
\hline 10 & $\begin{array}{l}\text { MICROCLIMA. } \\
\text { Garantizar el confort de los ambientes exteriores } \\
\text { mediante el control de las condiciones climáticas }\end{array}$ & exterior & $\begin{array}{l}\text { Evaluar si las edificaciones cuentan con estudios } \\
\text { de factores micro climáticos para ambientes } \\
\text { exteriores. }\end{array}$ & $\begin{array}{c}\text { Plan } \\
\text { maestro }\end{array}$ & $\begin{array}{l}\text { Plan maestro que incluya: } \\
\text { Evaluación de temperatura y confort térmico } \\
\text { Exposición solar (vista del cielo y sombreado) } \\
\text { Dirección, movimiento y velocidad del aire } \\
\text { Polvo y contaminación, Entorno acústico. }\end{array}$ \\
\hline 11 & $\begin{array}{l}\text { MATERIALES Y RECURSOS. } \\
\text { Gestionar la reducción de los residuos de la } \\
\text { construcción, promover su reciclaje y la reutilización } \\
\text { de los materiales }\end{array}$ & Eficiencia de recursos. & $\begin{array}{l}\text { Evaluar los planes de manejo de residuos } \\
\text { durante la construcción y todas las etapas del } \\
\text { ciclo de vida. }\end{array}$ & $\begin{array}{c}\text { Plan de } \\
\text { manejo } \\
\text { ambiental }\end{array}$ & $\begin{array}{l}\text { Plan de manejo para determinar qué se puede } \\
\text { renovar, reutilizar, reciclar o mantener. }\end{array}$ \\
\hline 12 & $\begin{array}{l}\text { DISEÑO INCLUSIVO. } \\
\text { Mejorar la accesibilidad de personas con } \\
\text { discapacidad y fomentar la inclusión, desde el } \\
\text { diseño, de infraestructura adecuada para mejorar la }\end{array}$ & & $\begin{array}{l}\text { Evaluar si los edificios cuentan con un plan para } \\
\text { implementar espacios inclusivos en su entorno } \\
\text { externo e interno. }\end{array}$ & $\begin{array}{c}\text { Plan } \\
\text { maestro }\end{array}$ & $\begin{array}{l}\text { Plan maestro con aspectos relevantes del diseño } \\
\text { inclusivo del entorno externo e interno del } \\
\text { edificio. }\end{array}$ \\
\hline
\end{tabular}

\section{Aplicar Indicadores Sostenibles propuestos para evaluar las edificaciones}

Finalmente, con los indicadores de mayor relevancia se realizó la evaluación sustentable de las tres viviendas seleccionadas, el nivel de calificación es: si cumple/no cumple, como se muestra en la tabla 4. 


\section{Tabla 4}

Evaluación sostenible urbana de tres edificaciones aplicando los indicadores

\section{propuestos}

\begin{tabular}{|c|c|c|c|c|}
\hline INDICADOR & \multirow{3}{*}{$\begin{array}{c}\text { UNIDAD DE } \\
\text { MEDIDA Y } \\
\text { PARÁMETRO DE } \\
\text { CUMPLIMIENTO }\end{array}$} & \multicolumn{3}{|c|}{ CONSTRUCCIONES/PARROQUIA } \\
\hline SOSTENIBLE & & Totoracocha & Cañaribamba & Yanuncay \\
\hline URBANO & & & $5+2$ & 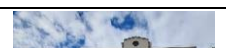 \\
\hline Obtención de & Certificado de & & & \\
\hline certificado & construcción & No cumple & No cumple & No cumple \\
\hline sostenible & sostenible & & & \\
\hline Estrategias para & $50 \%$ de agua lluvia & & & \\
\hline captar y & deberá ser utilizada & No cumple & No cumple & No cumple \\
\hline recolectar agua & para actividades no & & & \\
\hline lluvia & potables & & & \\
\hline Diseño inclusivo & Plan maestro & No cumple & No cumple & No cumple \\
\hline Proximidad a & Se encuentran a una & Sí cumple & Sí cumple & No cumple \\
\hline servicios de & distancia a pie de $1 / 4$ & $125 \mathrm{~m} \mathrm{de}$ & $180 \mathrm{~m}$ de distancia & $630 \mathrm{~m} \mathrm{de}$ \\
\hline transporte & de milla (400 metros) & distancia de un & de un transporte & distancia de un \\
\hline público & de un transporte & transporte & público de calidad. & transporte \\
\hline & público de calidad. & público de & & público de \\
\hline & & calidad. & & calidad. \\
\hline Tratamiento de & Gestionar residuos & & & \\
\hline Residuos & para tratar $75 \%$ a & No cumple & No cumple & No cumple \\
\hline Orgánicos & $100 \%$ de los residuos & & & \\
\hline & orgánicos. & & & \\
\hline
\end{tabular}

\section{Discusión}

De la evaluación realizada, en la cual se aplicó los parámetros de medida establecidos de los indicadores, se observó que las edificaciones no cumplen con la mayoría de estos, razón por la cual, no aportan con las características de un proceso de construcción sostenible, que sirva para orientar a la ciudad hacia un desarrollo sostenible, se observó principalmente que con relación al transporte público dos de las edificaciones cumplen con la distancia a pie (400 metros) para acceder a un transporte de calidad; sin embargo, la edificación que se encuentra emplazada en una parroquia en proceso de consolidación, 
no cumple con este parámetro pues los usuarios deben recorrer aproximadamente 630 metros para acceder al transporte público.

De lo analizado, concluimos que al día de hoy, las ciudades enfrentan grandes retos para lograr encaminarse hacia el desarrollo sostenible; y, a pesar de que uno de los aspectos más influyentes es lograr que la construcción, en su ciclo de vida, sea sostenible, todavía existen grandes desafíos para su cumplimiento, pues para determinar las condiciones de las edificaciones, se debe considerar, que están afectadas tanto por su entorno y sus características constructivas o de remodelación (Adamkiewicz et al., 2011).

El objetivo de este estudio fue explorar y proponer los indicadores más relevantes para que la ciudad mida su desempeño en relación con la construcción sostenible y determinar si hay un progreso hacia el cumplimiento de esta meta para conseguir el equilibrio anhelado.

\section{Conclusiones}

- En la ciudad de Cuenca, en el área urbana, actualmente el desarrollo de la construcción sostenible es casi nula, pues de la investigación se evidencia que, del universo total de bloques edificados, sólo el 0,00002\% (seis edificaciones) cuentan o están en proceso de certificarse como sostenibles.

- No existen políticas claras, por parte del ente regulador del espacio público, para fomentar o incentivar prácticas de sostenibilidad de la construcción en su ciclo de vida.

- Del análisis realizado a la bibliografía internacional sobre indicadores, detallada en esta investigación, encontramos que las herramientas LEED y BREEAM brindan un gran aporte sobre indicadores relacionados a medir la sostenibilidad urbana específica sobre la construcción sostenible.

- No existe en la región y en la localidad información de calidad y de fácil acceso, datos estadísticos y datos actualizados lo que dificulta la propuesta de indicadores para la localidad.

- Para la localidad, se muestran cinco indicadores relevantes: Obtención de un certificado de construcción sostenible, Estrategias para captar y recolectar agua de lluvia, Diseño inclusivo, Proximidad a servicios de transporte público y Tratamiento de Residuos Orgánicos. De la evaluación realizada a las tres edificaciones, se concluye que, para orientar a la ciudad hacia un desarrollo sostenible, se debe trabajar en fortalecer las políticas públicas que encaminen a los usuarios, constructores y promotores a que, en sus proyectos, desde la etapa 
de concepción, cumplan con prácticas enfocadas a la sostenibilidad, pues se evidencia que estas prácticas no están enquistadas en los actores mencionados.

\section{Agradecimientos}

El presente artículo es parte del trabajo de investigación y titulación del Programa de Maestría en Construcción con Mención en Administración de la Construcción Sustentable de la Universidad Católica de Cuenca, vinculados al Proyecto de Investigación: INDICADORES DE SOSTENIBILIDAD URBANA PARA LA CIUDAD DE CUENCA - ECUADOR, por ello agradecemos a todos y cada uno de los instructores pertenecientes a los grupos de investigación; Ciudad, Ambiente y Tecnología (CAT), y Sistemas embebidos y visión artificial en ciencias, Arquitectónicas, Agropecuarias, Ambientales y Automática (SEVA4CA), por los conocimientos e información brindados para la elaboración del trabajo.

\section{Referencias Bibliográficas}

Adamkiewicz, G., Zota, A. R., Patricia Fabian, M., Chahine, T., Julien, R., Spengler, J. D., \& Levy, J. I. (2011). Moving environmental justice indoors: Understanding structural influences on residential exposure patterns in low-income communities. American Journal of Public Health, 101(SUPPL. 1), 238-245. https://doi.org/10.2105/AJPH.2011.300119

Ahvenniemi, H., Huovila, A., Pinto-Seppä, I., \& Airaksinen, M. (2017). What are the differences between sustainable and smart cities? Cities, 60, 234-245. https://doi.org/10.1016/j.cities.2016.09.009

Batalhao, A. C. S., Teixeira, D., Martins, M. de F., Bellen, H. M. van, \& Caldana, A. C. F. (2019). Sustainability Indicators: Relevance, Public Policy Support and Challenges. Journal of Management and Sustainability, 9(2), 173-190. https://doi.org/10.5539/jms.v9n2p173

Bibri, S. E. (2018). A foundational framework for smart sustainable city development: Theoretical, disciplinary, and discursive dimensions and their synergies. Sustainable Cities and Society, 38(April), 758-794. https://doi.org/10.1016/j.scs.2017.12.032

BID. (2014). Cuenca Ciudad sostenible / Plan de acción. In Banco Interamericano de desarrollo.

Bustos, C., \& Chacón, G.-B. (2009). El desarrollo sostenible y la agenda 21 Sustainable Development, Agenda 21. Telos, 11(2), 164-181.

Commission on Environment and Development, R. (1987). General Assembly. In United 
Nations (Vol. 10). https://doi.org/10.1017/s0251107x00020617

Cumbre de la Tierra, de R. de J. (1992). La Conferencia de Naciones Unidas sobre el medio ambiente y el desarrollo. In Publicación de las Naciones Unidas Número de venta: S.93.I.8 (Issue 2327).

Escobar, J., \& Cuervo, Á. (2008). Validez De Contenido Y Juicio De Expertos: Una Aproximación a Su Utilización. Avances En Medición, 6(septiembre), 27-36.

Escurra, L. M. (1988). Cuantificación de la validez de contenido por criterio de jueces. $103-111$.

Galicia Alarcón, L., Balderrama Trapaga, J., \& Edel Navarro, R. (2017). Validez de contenido por juicio de expertos: propuesta de una herramienta virtual. Apertura: Revista de Innovación Educativa, 9(2), 42-53. https://doi.org/10.18381/ap.v9n2.993

Horman, M. J., Riley, D., Lapinski, A. R., Korkmaz, S., Pulaski, M. H., Magent, C. S., Luo, Y., Harding, N., \& Dahl, P. K. (2006). Delivering green buildings: Process improvements for sustainable construction. Journal of Green Building, 1(1), 123140. https://doi.org/10.3992/jgb.1.1.123

Instituto Nacional de Estadísticas y Censos [INEC]. (2010). Fascículo Provincial Azuay. Equipo de Comunicación y Análisis Del Censo de Población y Vivienda. INEC., $1(1), 8$.

Organización de las Naciones Unidas [ONU], O. (2000). Agenda 21 (2000). 21, 689 p. http://biblioteca.semarnat.gob.mx/janium/recursos/224844/Contenido/H\%25progra mas/23\%25Agenda\%2521.pdf

Organización de las Naciones Unidas [ONU], O. (2015). Objetivo 11. Ciudades y comunidades Sostenibles. United Nations, 1-2. https://www.undp.org/content/undp/es/home/sustainable-development-goals/goal11-sustainable-cities-and-communities.html

Quesada Molina, F. (2014). Métodos de evaluación sostenible de la vivienda: Análisis comparativo de cinco métodos internacionales. Revista Hábitat Sustentable, 4(1), $56-67$.

Quiroga, R. (2001). Indicadores de sostenibilidad ambiental y de desarrollo sostenible. In Cepal Eclac (Vol. 16). http://www.eclac.cl/publicaciones/xml/8/9708/lc11607e_ind.pdf

Serrano, A., Molina, F., Catalán, M., Mena, V., \& Valdez, D. (2015). Sobre La Evaluación De La Sostenibilidad De Materiales De Construcción. Arte y Sociedad. 
Revista de Investigación, 9.

Soffia, G. P. (2020). Los materiales de construcción su ausencia en la Certificación de edificio sustentable (CES), Chile. Arquitectura y Urbanismo, 41(2), 93-103.

Soto, C. M., \& Segovia, J. L. (2009). Intervalos de confianza asimétricos para el índice la validez de contenido: Un programa Visual Basic para la V de Aiken. Anales de Psicología, 25(1), 169-171. http://revistas.um.es/analesps

Terraza, H., Blanco, D. R., \& Vera, F. (2014). De ciudades emergentes a ciudades sostenibles. In Ediciones $A R Q$ (Vol. 32, Issue 1).

UN-Hábitat, Adrián Moredia Valek, Diego Perez, P. A. (2018). Housing at the center of the Sustainable Development Goals in Mexico. In UN-Habitat.

Valdez, D. S. (2021). ¿(In) sostenibles? Confrontando la sostenibilidad urbana a los "barrios pobres" dominicanos. Revista Invi, 36(101), 173-199.

Verma, P., \& Raghubanshi, A. S. (2018). Urban sustainability indicators: Challenges and opportunities. Ecological Indicators, 93(June 2020), 282-291. https://doi.org/10.1016/j.ecolind.2018.05.007

\section{Conflicto de intereses}

Los autores declaramos que no existe conflicto de intereses en relación con el artículo presentado.

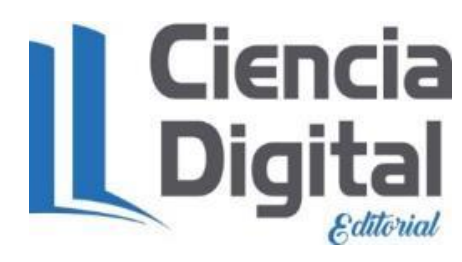




\section{Conciencia}

El artículo que se publica es de exclusiva responsabilidad de los autores y no necesariamente reflejan el pensamiento de la Revista Conciencia Digital.

\section{Ciencia \\ LDigital}

El artículo queda en propiedad de la revista y, por tanto, su publicación parcial y/o total en otro medio tiene que ser autorizado por el director de la Revista Conciencia Digital.
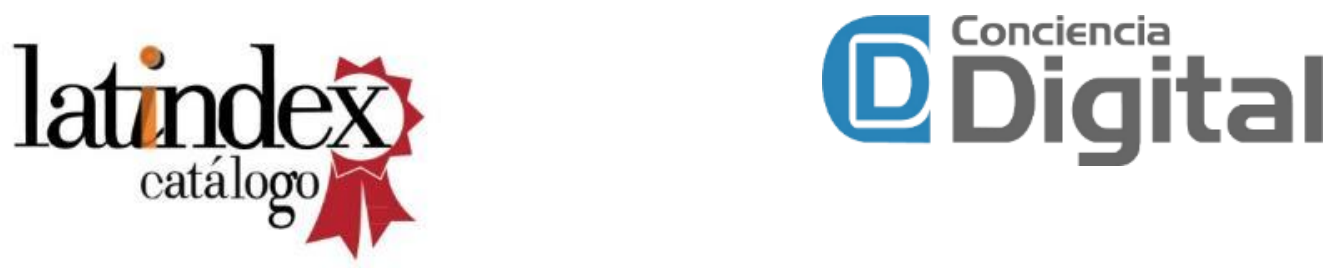

Indexaciones

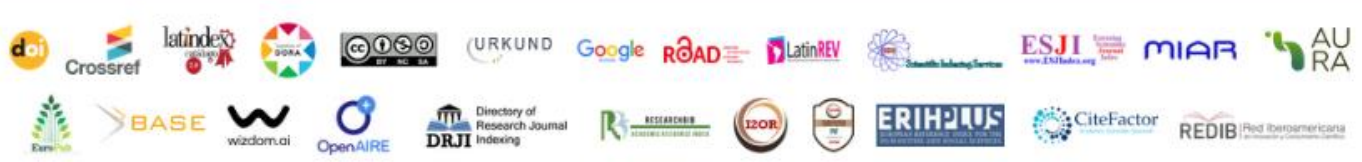

